\title{
Targeting Lipid Rafts, Seeking Cures for Diseases
}

\author{
Xin Wang* \\ ACURE Biotechnology, Inc., Ellicott City, Maryland, USA
}

Received: January 15, 2015; Accepted: January 22, 2015; Published: February 02, 2015

*Corresponding author: Xin Wang, ACURE Biotechnology, Inc., Ellicott City, Maryland, USA, Tel: 1-240-529-2819; E-mail: acurebiotech@gmail.com

\section{Editorial}

Lipid rafts are functional nanoscale microdomains enriched in cholesterol and sphingolipids, float in bilayer homogeneous lipids membrane in eukaryotic cells. Depending on anchored proteins through Glycosylphosphatidylinositol (GPI), or other lipids targeting modification, the microdomains of membrane play very important physiological roles in particular organs, such as bone and blood, cardiovascular, liver, brain etc. through intracellular membrane trafficking, cell signaling transduction and adaptation to microenvironment. For instance, ion channel proteins and membrane proteins anchoring in lipid rafts of cardiac myocytes govern the electrical-impulse, regulate polarizing of cells, balance metabolic resources, and stabilize the functional microdomains between the actin cytoskeleton and extracellular matrix. Most recently, targeting lipid rafts modification has become a unified strategy in immunotherapy of cancer, diabetes, cardiovascular diseases and Regenerative medicine for replacing neuron and human coronary artery smooth muscle cell. This editorial will summarize my original research in past fifteen years. These are the foundation of ACURE Biotechnology, Inc., company.

Myristoylation and palmitoylation are two important coand post-translational modifications for proteins to target lipid rafts. G protein alpha subunits and a group of Src-related protein tyrosine kinases are the proteins with both $\mathrm{N}$-myristoylation and palmitoylation. A variety of viral proteins such as Gag proteins also possess same modification. Luckily, we discovered a novel gene having same $\mathrm{N}$-terminal motif in post synaptic lipid rafts that is brain specific, but highly expressed in acute leukemia-BAALC (brain and acute leukemia, cytoplasmic) [1]. Our study confirmed the activity of $\mathrm{N}$-terminal myristoylation and palmitoylation of BAALC 1-6-8 through mutation study. Emerging evidence suggest that BAALC expression stops differentiation of myeloid [2]. Clinical data underlined that BAALC is a suitable marker to prognosis outcome of a sub-group acute myeloid leukemia [3]. However, the detailed mechanism of BAALC 1-6-8 in hematopoietic system remains unclear. The BAALC knockout mouse seems do not survive, our future study will employ knock-in or transgenic animal models, and utilize other platforms to decipher the mechanism of BAALC in introduction and maintenance of stemness in oncology (hematology), uncover the regulation rules of expression of BAALC and expression pattern of its diverse isoforms in leukemia patients, explore the environmental risk factors to develop preventive medicine for leukemia.

The components and ratio of lipids species assembled into biomembrane is another essential factor contributing to the specific behaviors of lipid rafts. From two lipid metabolism genes mutation models, we understood that the nature of biomembrane are very different between mammals and Drosophila. NeutralCeramidase (N-CDase) nude mice do not have significant phenotype, even the N-CDase nude MEFs only show around $10 \%$ weak cytotoxic toleration as compared to wild type MEFs by FACS analysis (unpublished data), whereas Drosophila N-CDase knockout is lethal [4]. In addition, the outcome of impairment of lipid metabolism and membrane organization affects profoundly different on survival and adaptation responses between mouse and fly: most of Ceramide Transfer Protein (Cert) nude mice die from organogenetic defects at the stage of embryo 11. 5 days without any significant changes in fluidity of membrane [5]. Interesting enough, Cert nude flies fully develop to adulthood with around $35 \%$ increase of fluidity of membrane, $33 \%$ and $40 \%$ increase of cellular glucose and modification proteins, respectively, which resulted in shorter lifespan to flies [6].

The biological aging is the most risk factor for human diseases. The alteration of the lipid metabolism and cholesterol homeostasis contribute to cardiovascular diseases caused by aging of blood vessels, Alzheimer's disease character by neuron degeneration, and so on. Up to date, the most accepted events that are demonstrated to influence the aging process are energy metabolism: Caloric restriction, insulin pathways and electron transport chains, all mainly occur in mitochondria. In our Cert mutant mouse model, ceramide accumulation in ER results in mild ER adaption response to the stress, but severe mitochondria dysfunction. With the altered sphingolipids components in plasma membrane and organelles, developmental defects of heart in this Cert mutant mouse lead embryo to die from heart failure. As the primary researcher of the mouse model, I hypothesize that the biological aging process in cells due to dysfunction of mitochondria is the major cause of organogenetic failure in Cert null mice. Our next study will explore how lipid metabolism play a role in aging process with Cert mutant mouse model, we will develop and discover some compounds involved in lipid metabolism that may able to rescue the developmental defect of cardiovascular that have high morbidity in new births. 
Glycolipids and glycoprotein's (glycosylated proteins) are very important components in functional microdomains of membrane in mammals. Especially, in Immune system, all kinds of immunoglobulins are glycoproteins. It is reported that Cert expression decreases in Triple Negative Breast Cancer (TNBC), an aggressive cancer type in metastasis [7]. Consistent with the recent discovery [8]: Lipid rafts and its associated actin cytoskeleton contribute to the localization and function of $\mathrm{ABC}$ transporter, the marker of side population in flow cytometry, our unpublished data with head and neck Squamous cell carcinoma (HNSCC) suggest that hypoxia induces the characteristic of cancer stem cell, hormone and growth factor enhance hypoxic response of HNSCC, which are probably the reason of resistance to chemotherapy for cancers. Besides the sphingomyelin, ceramide is also the precursor for biosynthesis of glucosylceramide, which reduce the sensitivity of cancer cells to drugs and involve in maintaining features of cancer stem cell. In our Cert mutant mouse model, the glucosylceramide is probably overproduced by ceramide accumulation. In addition, our unpublished data shows that the expression level of Tenascin C (TNC), a glycoprotein normally found abundant in extracellular matrix, associate with hypoxia in aggressive HNSCC cell lines. With these previous work, our company will study the mechanism of chemotherapy resistant involved by lipid metabolism with Cert mutant mouse, and develop drugs to neutralize the toleration to anti-cancer of Glycolipids.

In infectious diseases, both bacteria and virus alter the lipid metabolism during the replication and secretion. Secretion of cytokines in inflammation and production of antibodies to specific stimulation of antigens need rearrangement of lipid rafts, progression of endocytosis and exocytosis. For the interaction between ligands and receptors, internalization of membrane for signaling transduction need particular functional lipid rafts clusters. For example, in chronic Hepatitis C virus (HCV) infection, lipoproteins and membrane glycoprotein's combined with virus in serum to moderate immune response from the host, lipoproteins also regulate the entry of virus into hepatocytes. The disorder of lipid metabolism causes the liver cirrhosis, even Hepatocellular carcinoma [9]. Our recent study established an angiogenic animal model through intervention and induction of periodontitis [10]. We will study the mechanism of vasculature along with bone resorption in inflammatory microenvironment, seek stem cell therapy in treatment of cardiovascular, bone resorption and bone metastasis of cancer.

Taken together, our company will conduct innovative research on targeting lipid functional microdomains, lipid rafts; discover stem cell-related therapeutic approaches for cancer, chronic infectious diseases, cardiovascular diseases, osteoporosis, Alzheimer's disease and other aging associated diseases. Our scientific designing, and ethical clinical trials will provide reliable data for the improvement of human health.

\section{References}

1. Wang X, Tian QB, Okano A, Sakagami H, Moon IS, Kondo H, et al. BAALC 1-6-8 protein is targeted to postsynaptic lipid rafts by its $\mathrm{N}$-terminal myristoylation and palmitoylation, and interacts with a, but not $\mathrm{b}$, subunit of $\mathrm{Ca} 2+$ /calmodulin-dependent protein kinase II. J Neurochem. 2005; 92(3): 647-659.

2. Heuser M, Berg T, Kuchenbauer F, Lai CK, Park G, Fung S, et al. Functional role of BAALC in leukemogenesis. Leukemia. 2012; 26(3): 532-536. doi: 10.1038/leu.2011.228.

3. Weber S, Alpermann T, Dicker F, Jeromin S, Nadarajah N, Eder C, et al. BAALC expression: a suitable marker for prognostic risk stratification and detection of residual disease in cytogenetically normal acute myeloid leukemia. Blood Cancer J. 2014; 4: e173. doi: 10.1038/ bcj.2013.71.

4. Changqing Yuan, Raghavendra Pralhada Rao, Nahid Jesmin, Takeshi Bamba, Kunio Nagashima, Alberto Pascual, et al. CDase is a panCeramidase in Drosophila. Mol Biol Cell. 2011; 22(1): 33-43. doi: 10.1091/mbc.E10-05-0453.

5. Wang X, Rao RP, Kosakowska-Cholody T, Masood MA, Southon E, Zhang HL, et al. Mitochondrial degeneration and not apoptosis is the primary cause of embryonic lethality in ceramide transfer protein mutant mice. J Cell Biol. 2009; 184(1): 143-158. doi: 10.1083/ jcb.200807176.

6. Rao RP, Yuan C, Allegood JC, Rawat SS, Edwards MB, Wang X, et al. Ceramide transfer protein function is essential for normal oxidative stress response and lifespan. Proc Natl Acad Sci USA. 2007; 104(27): 11364-11369.

7. Heering J, Weis N, Holeiter M, Neugart F, Staebler A, Fehm TN, et al. Loss of the ceramide transfer protein augments EGF receptor signaling in breast cancer. Cancer Res. 2012; 72(11): 2855-2866. doi: 10.1158/0008-5472.CAN-11-3069.

8. Kok JW, Klappe K, Hummel I. The Role of the Actin Cytoskeleton and Lipid Rafts in the Localization and Function of the ABCC1 Transporter. Advances in Biology. 2014; 105898.

9. Rojas A, del Campo JA, Maraver M, Aparcero R, Garcia-Valdecasas M, Diago M, et al. Hepatitis C virus infection alters lipid metabolism depending on IL28B polymorphism and viral genotype and modulates gene expression in vivo and in vitro. Journal of Viral Hepatitis. 2014; 21(1): 19-24. doi: 10.1111/jvh.12209.

10.Zhang ZY, Ge XJ, Zheng WX, Chen RZ, Wang X. VEGFA And IL17 Expression Reveals Their Potential Functional Crosstalk in Periodontitis Rats-A New Animal Model for Angiogenesis Study. Journal of Microbiology \& Experimentation. 2014; 1(4). 